\title{
One-Parameter GHG Emission Policy with R\&D-based Growth
}

\author{
Tapio Palokangas \\ University of Helsinki and HECER
}

Discussion Paper No. 366

May 2013

ISSN 1795-0562

HECER - Helsinki Center of Economic Research, P.O. Box 17 (Arkadiankatu 7), FI-00014 University of Helsinki, FINLAND, Tel +358-9-191-28780, Fax +358-9-191-28781, E-mail info-hecer@helsinki.fi, Internet www.hecer.fi 


\title{
One-Parameter GHG Emission Policy for Endogenously-Growing Regions*
}

\begin{abstract}
This document examines the GHG emission policy of regions which use land, labor and emitting inputs in production and enhance their productivity by devoting labor to R\&D. The problem is to organize common emission policy, if the regions cannot form a federation with a common budget and the policy parameters must be uniform for all regions. The results are the following. An agreement of the self-interested central planner that allocates emission caps in fixed proportion to past emissions (i.e. grandfathering) leads to the Pareto optimum, decreasing emissions and promoting R\&D and economic growth.
\end{abstract}

JEL Classification: 041, H23, F15, Q53

Keywords: GHG emissions; endogenous growth; lobbying; emission caps

Tapio Palokangas

Department of Political and Economic Studies

University of Helsinki

P.O. Box 17

FI-00014 University of Helsinki

FINLAND

e-mail: tapio.palokangas@helsinki.fi

${ }^{*}$ The main ideas of this paper were finalized during my several visits to IIASA in 2011 and 2012. The author thanks IIASA for hospitability during that time. 


\section{Introduction}

This document examines a number of regions that produce the final good from land, labor and an emitting input and enhance their productivity by devoting labor to R\&D. The regions establish a central planner that decides how much each region can emit greenhouse gases (GHGs). Because the regions do not form a federation, the central planner is self-interested (i.e. subject to lobbying) and has no budget of its own. Furthermore, the central planner can use only one policy parameter that must be uniformly applied to all regions. In this framework, it is instructive to compare the cases of laissez-faire, the Pareto optimum and lobbying equilibrium.

It has been common in environmental economics to consider abatement in a two-sector framework where one sector produces a final good, but the other sector alleviates the use of natural resources (cf. Xepapadeas 2005, chapter 4.3). The problem of environmental policy is then basically static: it answer the question of how resources could be optimally allocated between the sectors. Because that approach ignores the long traces that environmental policy may cause for the economic growth of countries, this document examines emissions in a R\&D-based growth model.

Haurie et al. (2006) examine a negotiation game where the regions talk over an international agreement on their use of GHGs to foster their economic development. They show that if GHGs in the atmosphere are exogenously constrained, then there is a Pareto optimum in these talks. Böringer and Lange (2005) and Mackenzie et al. (2008) consider emissions-based allocation rules for which the basis of allocation is updated over time. They show that if the emission cap is absolute, then grandfathering schemes - which allocate allowances proportionally to past emissions - lead to the first-best. This document extends the analysis of these papers as follows. First, the policy maker in the coalition is self-interested, being subject to lobbying from the regions. Second, the international emission cap is endogenously determined 
by the same bargaining between the coalition members and the policy maker.

Jouvet et al. (2008) incorporate externality through pollution in an overlapping-generations (OLG) model, showing that the optimal growth path can be decentralized only with lump-sum transfers and a market for GHG permits. All permits should then be auctioned, which rules out all grandfathering practises. Jouvet et al. (2008) explain these results as follows: grandfathering practices cause a distortion by raising the return on investment, but the lump-sum provision of pollution rights to households does not distort anything. In contrast, this document considers the coordination of environmental policy through the design of a policy maker with no budget. It is instructing to see whether grandfathering schemes distort in that setting.

Palokangas (2009) considers emission policy with a self-interested central planner in a coalition of identical regions. That paper however assumes, rather unrealistically, that technology and primary resources are similar in all regions and that the central planner can negotiate over different emission caps with different regions. In this document, that assumption is relaxed: the central planner has only one policy parameter - the proportion of grandfathering in allocating emissions caps - that must be uniformly applied to all regions. Sections 2 presents the structure of the economy and section 3 constructs the model for a single region. Sections 4,5 and 6 examine the cases of laissez-faire, the Pareto optimum and lobbying, respectively. It is shown that a one-parameter grandfathering agreement is self-enforcing (cf. Haurie et al. 2006): no region has incentives to break it.

\section{The economy}

The economy contains a large number (a "continuum") of regions placed evenly in the limit $[0,1]$. Each region $j \in[0,1]$ supplies land $A_{j}$ and labor $L_{j}$ inelastically, and devotes $l_{j}$ units of labor to production and the remainder

$$
z_{j}=L_{j}-l_{j}
$$


to R\&D. There exists an emitting input called energy the extraction costs of which are ignored, for simplicity. It is assumed that local emissions are proportional to the use of energy, $m_{j}$, in each region $j$. Pollution $m$ is a linearly homogeneous function $M$ of the emissions of all regions $j \in[0,1]$ :

$$
m=M\left(m_{j} \mid j \in[0,1]\right), \quad M \text { homogeneous of degree one. }
$$

All regions produce the same consumption good from land, labor and energy. That good is chosen as the numeraire, for simplicity.

To enable that the regions can increase their efficiency and consequently grow at different rates in a stationary-state equilibrium, we eliminate

- the terms-of-trade effect by the assumption that all regions produced the same internationally-traded good, and

- international capital movements by the assumption that all regions share the same constant rate of time preference, $\rho$.

On the assumption of perfect markets, each region $j \in[0,1]$ behaves as if there were a single agent (hereafter called region $j$ ) that controls fully the resources in that region. This document ignores free riding, for simplicity: all regions $j \in[0,1]$ are committed to common emission policy.

\section{Single region $j \in[0,1]$}

\subsection{Production}

When region $j$ develops a new technology, it increases its productivity by constant proportion $a_{j}>1$. The level of productivity in region $j$ is then equal to $a_{j}{ }^{\gamma_{j}}$, where $\gamma_{j}$ is its serial number of technology. The innovation of new technology in region $j$ increases $\gamma_{j}$ by one.

Region $j$ produces its output $y_{j}$ from land $A_{j}$, labor $l_{j}$ and energy $m_{j}$. It is assumed that local emissions, which are proportional to energy input 
$m_{j}$, harm production by spoiling the quality of the product. ${ }^{1}$ It is futhermore assumed that labor $l_{j}$ and energy $m_{j}$ form a composite input $\phi^{j}\left(l_{j}, m_{j}\right)$ through CES technology, but otherwise there is Cobb-Douglas technology: ${ }^{2}$

$y_{j}=a_{j}^{\gamma_{j}} f^{j}\left(l_{j}, m_{j}\right) m_{j}^{-\beta}, f^{j}\left(l_{j}, m_{j}\right) \doteq A_{j}^{1-\alpha_{j}} \phi^{j}\left(l_{j}, m_{j}\right)^{\alpha_{j}}, \quad 0<\alpha_{j}<1, \quad \beta>0$,

$f_{l}^{j}>0, f_{m}^{j}>0, \phi_{l}^{j}>0, \phi_{m}^{j}>0, \phi_{l l}^{j}<0, \phi_{m m}^{j}<0, \phi_{l m}^{j}>0$,

where the subscripts $l$ and $m$ denote the partial derivative of the function with respect to $l_{j}$ and $m_{j}$, respectively, $a_{j} \gamma_{j}$ is total factor productivity, $\alpha_{j}$ a parameter and $\beta$ is the constant elasticity of output with respect to emissions $m_{j}$. The higher $\beta$, the more local emissions $m_{j}$ harm local production.

When the markets are perfect in region $j$, one can interpret $1-\alpha_{j}$ as the expenditure share of land and $\alpha_{j}$ that of labor and energy taken together. Noting (3), the expenditure shares of energy and labor in production are

$$
\begin{aligned}
& \frac{m_{j} f_{m}^{j}\left(l_{j}, m_{j}\right)}{f^{j}\left(l_{j}, m_{j}\right)}=\alpha_{j} \frac{m_{j} \phi_{m}^{j}\left(l_{j}, m_{j}\right)}{\phi^{j}\left(l_{j}, m_{j}\right)}=\alpha_{j} \frac{\phi_{m}^{j}\left(l_{j} / m_{j}, 1\right)}{\phi^{j}\left(l_{j} / m_{j}, 1\right)} \doteq \xi^{j}\left(\frac{l_{j}}{m_{j}}\right) \in\left(0, \alpha_{j}\right), \\
& \frac{l_{j} f_{l}^{j}\left(l_{j}, m_{j}\right)}{f^{j}\left(l_{j}, m_{j}\right)}=\alpha_{j} \frac{l_{j} \phi_{l}^{j}\left(l_{j}, m_{j}\right)}{\phi^{j}\left(l_{j}, m_{j}\right)}=\alpha_{j}\left[1-\frac{m_{j} \phi_{m}^{j}\left(l_{j}, m_{j}\right)}{\phi^{j}\left(l_{j}, m_{j}\right)}\right]=\alpha_{j}-\xi^{j}\left(\frac{l_{j}}{m_{j}}\right) \\
& \in\left(0, \alpha_{j}\right) .
\end{aligned}
$$

Because the composite input $\phi^{j}\left(l_{j}, m_{j}\right)$ is a CES function, one obtains

$$
\left(\xi^{j}\right)^{\prime}\left(\frac{l_{j}}{m_{j}}\right)=\frac{d \xi^{j}}{d\left(l_{j} / m_{j}\right)} \begin{cases}>0 & \text { for } 0<\sigma_{j}<1, \\ <0 & \text { for } \sigma_{j}>1,\end{cases}
$$

where $\sigma_{j}$ is the constant elasticity of substitution between inputs $l_{j}$ and $m_{j}$.

\subsection{Research and development (R\&D)}

An increase in productivity in region $j, a_{j}{ }^{\gamma_{j}}$ [cf. (3)], depends on labor devoted to $\mathrm{R} \& \mathrm{D}, z_{j}$, in that region: the probability that input $z_{j}$ leads to

\footnotetext{
${ }^{1}$ Without this assumption, region $j$ would use an indefinitely large amount of energy in the case of laissez-faire (cf. section 4 ).

${ }^{2}$ The use of a general production function $y_{j}=a_{j}^{\gamma_{j}} F\left(A_{j}, l_{j}, m_{j}\right)$ would excessively complicate the analysis.
} 
development of a new technology with a jump from $\gamma_{j}$ to $\gamma_{j}+1$ in a small period of time $d \theta$ is given by $\lambda_{j} z_{j} d \theta$, while the probability that input $z_{j}$ remains without success is given by $1-\lambda_{j} z_{j} d \theta$, where $\lambda_{j}>0$ is a constant. Noting (1), this defines a Poisson process $\chi_{j}$ with

$$
d \chi_{j}= \begin{cases}1 & \text { with probability } \lambda_{j} z_{j} d \theta, \\ 0 & \text { with probability } 1-\lambda_{j} z_{j} d \theta, \quad z_{j}=L_{j}-l_{j},\end{cases}
$$

where $d \chi_{j}$ is the increment of the process $\chi_{j}$.

\subsection{Preferences}

All regions have the same preferences: the expected utility of region $j \in[0,1]$ starting at time $T$ is given by

$$
E \int_{T}^{\infty} c_{j} m^{-\delta} e^{-\rho(\theta-T)} d \theta, \quad \delta>0, \quad \rho>0,
$$

where $E$ is the expectation operator, $\theta$ time, $c_{j}$ consumption in region $j, \rho$ the constant rate of time preference and $\delta$ the constant elasticity of temporary utility with respect to economy-wide emissions $m$. The lower $\rho$, the more patient the regions are. Total pollution $m$ decreases welfare in all regions $j \in[0,1]$, but a single region is so small that it ignores this dependence. The higher $\delta$, the more pollution $m$ is disliked.

\section{Laissez-faire}

Because all regions $j \in[0,1]$ produce the same consumption good, then, without GHG emissions management, each region $j$ consumes what it produces, $c_{j}=y_{j}$. Noting (3) and $c_{j}=y_{j}$, the expected utility of the region starting at time $T,(7)$, becomes

$$
\Upsilon^{j}=E \int_{T}^{\infty} y_{j} m^{-\delta} e^{-\rho(\theta-T)} d \theta=E \int_{T}^{\infty} a_{j}^{\gamma_{j}} f^{j}\left(l_{j}, m_{j}\right) m_{j}^{-\beta} m^{-\delta} e^{-\rho(\theta-T)} d \theta .
$$

Assume for a while that energy input $m_{j}$ is held constant. Region $j$ then maximizes its expected utility (8) by its labor devoted to production, $l_{j}$, 
subject to its technological change (6), given pollution $m$. The solution of this maximization is the following (cf. Appendix A):

Proposition 1 The expected utility of region $j$ is

$$
\Upsilon^{j}=m^{-\delta} \Pi^{j}\left(\gamma_{j}, m_{j}, T\right), \text { for which } \frac{\partial \Pi^{j}}{\partial m_{j}}=\frac{\Pi^{j}}{m_{j}}\left[\xi^{j}\left(\frac{l_{j}}{m_{j}}\right)-\beta\right] \text {. }
$$

Region $j$ chooses its labor input $l_{j}$ so that

$$
\frac{\left(a_{j}-1\right) \lambda_{j} l_{j}}{\rho+\left(1-a_{j}\right) \lambda_{j}\left(L_{j}-l_{j}\right)}=\alpha_{j}-\xi^{j}\left(\frac{l_{j}}{m_{j}}\right) .
$$

In the presence of laissez-faire, region $j$ can optimally determine its energy input $m_{j}$ as well: it maximizes the value of its program, $\Upsilon^{j}$, by $m_{j}$. Given (9), this leads to the first-order condition

$$
\frac{\partial \Upsilon^{j}}{\partial m_{j}}=m^{-\delta} \frac{\partial \Pi^{j}}{\partial m_{j}}=m^{-\delta} \frac{\Pi^{j}}{m_{j}}\left[\xi^{j}\left(\frac{l_{j}}{m_{j}}\right)-\beta\right]=0 \quad \text { and } \quad \xi^{j}\left(\frac{l_{j}}{m_{j}}\right)=\beta .
$$

The second-order condition of the maximization is given by

$$
\frac{\partial^{2} \Upsilon^{j}}{\partial m_{j}^{2}}=-\underbrace{m^{-\delta} \frac{\Pi^{j}}{m_{j}}}_{+}\left(\xi^{j}\right)^{\prime} \underbrace{\frac{l_{j}}{m_{j}^{2}}}_{+}<0 \text { and }\left(\xi^{j}\right)^{\prime}>0 .
$$

Given this and (5), labor and energy are gross complements, $0<\sigma_{j}<1$, and $\left(\xi^{j}\right)^{\prime}>0$ holds true everywhere. From this, (10) and (11) it follows that

$$
\frac{\left(a_{j}-1\right) \lambda_{j} l_{j}^{L}}{\rho+\left(1-a_{j}\right) \lambda_{j}\left(L_{j}-l_{j}^{L}\right)}=\alpha_{j}-\beta, \quad \xi^{j}\left(\frac{l_{j}^{L}}{m_{j}^{L}}\right)=\beta \text { with }\left(\xi^{j}\right)^{\prime}>0,
$$

where the superscript $L$ denotes the laissez-faire equilibrium.

Finally, the following result is proven in Appendix B:

Proposition 2 The more emissions harm locally (i.e. the higher $\beta$ ), the less there are emissions $m_{j}^{L}, d m_{j}^{L} / d \beta<0$, and the more there is $R \& D$ (i.e. the higher $\left.z_{j}^{L}\right), d z_{j}^{L} / d \beta>0$.

Because technological change generated by $R \& D$ decreases the need for polluting energy, there are incentives to perform R\&D. 


\section{The Pareto optimum}

Grandfathering means that emission caps have a base that is determined by the history, but updated over time. In models with discrete time, that base would be calculated by a moving average of past emissions. In the qualityladders model of this document where time is continuous, the base is specified as follows. The central planner sets the pollutant caps in fixed proportion $\varepsilon$ to the energy input of that region under previous technology, $\widehat{m}_{j}$ :

$$
m_{j} \leq \varepsilon \widehat{m}_{j} \text { for } j \in[0,1] \text { and } \varepsilon>0 .
$$

If the current number of technology is $\gamma_{j}$, then the allocation base $\widehat{m}_{j}$ is calculated by energy input under previous technology $\gamma_{j}-1$ (cf. subsection 3.1). If the central planner tightens emission policy by decreasing $\varepsilon$ below one, then the constraint (13) becomes binding for all regions $j \in[0,1]$. Because the function $M$ in (2) is linearly homogeneous, one then obtains:

$$
m_{j}=\varepsilon \widehat{m}_{j} \text { for } j \in[0,1], \quad m=\varepsilon \widehat{m}, \quad \widehat{m} \doteq M\left(\widehat{m}_{j} \mid j \in[0,1]\right) .
$$

In the grandfathering scheme, there is thus only one policy parameter $\varepsilon$.

Because all regions $j \in[0,1]$ produce the same consumption good, total consumption is equal to total production, $\int_{0}^{1} c_{j} d j=\int_{0}^{1} y_{j} d j$. To construct the Pareto optimum, let us introduce a benevolent central planner that maximizes the welfare of the representative agent of the economy, $\mathcal{W}$. Given (7), (8), (9) and $\int_{0}^{1} c_{j} d j=\int_{0}^{1} y_{j} d j$, that welfare is

$$
\begin{aligned}
\mathcal{W} & \doteq \int_{0}^{1}\left[E \int_{T}^{\infty} c_{j} m^{-\delta} e^{-\rho(\theta-T)} d \theta\right] d j=E \int_{T}^{\infty}\left(\int_{0}^{1} c_{j} d j\right) m^{-\delta} e^{-\rho(\theta-T)} d \theta \\
& =E \int_{T}^{\infty}\left(\int_{0}^{1} y_{j} d j\right) m^{-\delta} e^{-\rho(\theta-T)} d \theta=E \int_{T}^{\infty}\left(\int_{0}^{1} y_{j} m^{-\delta} e^{-\rho(\theta-T)} d \theta\right) d j \\
& =\int_{0}^{1} \Upsilon^{j} d j=m^{-\delta} \int_{0}^{1} \Pi^{j}\left(\gamma_{j}, m_{j}, T\right) d j
\end{aligned}
$$

which should be maximized by the policy parameter $\varepsilon$. Given (9) and (14), 
this leads to the first-order conditions

$$
\begin{aligned}
0 & =\frac{d \mathcal{W}}{d \varepsilon}=m^{-\delta} \int_{0}^{1} \frac{\partial \Pi^{j}}{\partial m_{j}} \underbrace{\frac{\partial m_{j}}{\partial \varepsilon}}_{=\widehat{m}_{j}} d j-\delta m^{-\delta-1} \underbrace{\frac{\partial m}{\partial \varepsilon}}_{=\widehat{m}} \int_{0}^{1} \Pi^{j} d j \\
& =m^{-\delta}\left[\int_{0}^{1} \frac{\partial \Pi^{j}}{\partial m_{j}} \widehat{m}_{j} d j-\delta \frac{\widehat{m}}{m} \int_{0}^{1} \Pi^{j} d j\right] \\
& =m^{-\delta}\left\{\int_{0}^{1} \Pi^{j}\left[\xi^{j}\left(\frac{l_{j}}{m_{j}}\right)-\beta\right] \frac{\widehat{m}_{j}}{m_{j}} d j-\delta \frac{\widehat{m}}{m} \int_{0}^{1} \Pi^{j} d j\right\} \\
& =m^{-\delta} \int_{0}^{1} \Pi^{j}\left\{\left[\xi^{j}\left(\frac{l_{j}}{m_{j}}\right)-\beta\right] \frac{\widehat{m}_{j}}{m_{j}}-\delta \frac{\widehat{m}}{m}\right\} d j .
\end{aligned}
$$

In the stationary state, all inputs $\left(l_{j}, m_{j}\right)$ for all regions $j \in[0,1]$ must be constant. Once the economy attains the stationary state, the emissions under the previous and current technology become equal: $\widehat{m}=m$ and $\widehat{m}_{j}=m_{j}$ for $j \in[0,1]$. Plugging these conditions and into (16) yields

$$
0=m^{-\delta} \int_{0}^{1} \Pi^{j}\left[\xi^{j}\left(\frac{l_{j}}{m_{j}}\right)-\beta-\delta\right] d j .
$$

Because the expected utilities $\Pi^{j}$ for $j \in[0,1]$ are random variables, then, given (17), the only possible stationary state is

$$
\xi^{j}\left(\frac{l_{j}}{m_{j}}\right)=\beta+\delta \text { for } j \in[0,1] .
$$

The equilibrium conditions (10) for the regions $j \in[0,1]$ as well as those (18) for the central planner can be written as

$$
\xi^{j}\left(\frac{l_{j}^{P}}{m_{j}^{P}}\right)=\beta+\delta, \quad \frac{(a-1) \lambda_{j} l_{j}^{P}}{\rho+(1-a) \lambda_{j}\left(L_{j}-l_{j}^{P}\right)}=\alpha_{j}-\beta-\delta,
$$

where the superscript $P$ denotes the Pareto optimum equilibrium.

The comparison of (19) with (12) shows that the introduction of a benevolent central planner increases the parameter $\beta$ up to $\beta+\delta$ in the system. Thus, Proposition 2 has the following corollary:

Proposition 3 A shift from laissez-faire to the Pareto optimum decreases emissions, $m_{j}^{P}<m_{j}^{L}$, and increases $R \& D, z_{j}^{P}>z_{j}^{L}$. 
The introduction of a benevolent central planner internalizes the negative externality through emissions. This increases incentives to perform R\&D. With the uniform proportionality rule $\varepsilon$, all regions face the same marginal benefits from pollutants via allocation in subsequent periods. In contrast to Böringer and Lange (2005), the regulatory cap $m^{P}$ is not exogenous but endogenously determined.

\section{Regulation}

In this section, regions $j \in[0,1]$ lobby the central planner over the policy parameter $\varepsilon$. Following Grossman and Helpman (1994), it is assumed that the central planner has its own interests and collects political contributions $R_{j}$ from regions $j \in[0,1]$. This is a common agency game, the order of which is then the following (cf. Grossman and Helpman 1994, and Dixit et al. 1997). First, the regions $j \in[0,1]$ set their political contributions $R_{j}$ conditional on the central planner's prospective policy $\varepsilon$. Second, the central planner sets its policy $\varepsilon$ and collects the contributions from the regions. Third, the regions maximize their utilities. This game is solved in reverse order: Subsection 6.1 considers the equilibrium of the regions and 6.2 the political equilibrium.

\subsection{Optimal program}

Region $j$ pays its political contributions $R_{j}$ to the central planner. It is assumed, for simplicity, that the central planner consists of civil servants who inhabit regions $j \in[0,1]$ evenly. Thus, the regions gets an equal share $R$ of total contributions,

$$
R \doteq \int_{0}^{1} R_{j} d j / \int_{0}^{1} d k=\int_{0}^{1} R_{j} d j .
$$

Noting the production function (3), consumption in region $j$ is then

$$
c_{j}=y_{j}+R-R_{j}=a_{j}^{\gamma_{j}} f^{j}\left(l_{j}, m_{j}\right) m_{j}^{-\beta}+R-R_{j},
$$


where $y_{j}$ is income from production and $R-R_{j}$ net revenue from political contributions in region $j$. Noting (21), the expected utility of region $j$ starting at time $T,(7)$, becomes

$$
\Theta^{j}=E \int_{T}^{\infty}\left[a_{j}^{\gamma_{j}} f^{j}\left(l_{j}, m_{j}\right) m_{j}^{-\beta}+R-R_{j}\right] m^{-\delta} e^{-\rho(\theta-T)} d \theta .
$$

Region $j$ maximizes its expected utility (22) by its labor devoted to production, $l_{j}$, subject to technological change in the region, (6), given the emission cap $m_{j}$, pollution $m$ and political contributions $R_{j}$ and $R$. The solution for this optimal program is the function (cf. Appendix C)

$$
\begin{aligned}
& \Theta^{j}\left(m_{j}, m, R, R_{j}, \gamma_{j}\right), \quad \frac{\partial \Theta^{j}}{\partial m_{j}}=m^{-\delta} \frac{\Gamma^{j}\left(\gamma_{j}, m_{j}, T\right)}{m_{j}}\left[\xi^{j}\left(\frac{l_{j}}{m_{j}}\right)-\beta\right], \\
& \frac{\partial \Theta^{j}}{\partial m}=-\delta m^{-\delta-1}\left(\Gamma^{j}+\frac{R-R_{j}}{\rho}\right), \quad-\frac{\partial \Theta^{j}}{\partial R_{j}}=\frac{\partial \Theta^{j}}{\partial R}=\frac{m^{-\delta}}{\rho},
\end{aligned}
$$

where $\Gamma^{j}$ is the expected value of the flow of output for region $j$, which is a random variable, and $l_{j}^{*}$ is the optimal labor input in production for which

$$
\frac{\left(a_{j}-1\right) \lambda_{j} l_{j}^{*}}{\rho+\left(1-a_{j}\right) \lambda_{j}\left(L_{j}-l_{j}^{*}\right)}=\alpha_{j}-\xi^{j}\left(\frac{l_{j}^{*}}{m_{j}}\right) .
$$

\subsection{The political equilibrium}

Because each region $j$ affects the central planner by its contributions $R_{j}$, its contribution schedule depends on the central planner's policy $\varepsilon$ [cf. (20)]:

$$
R_{j}(\varepsilon) \text { for } j \in[0,1], \quad R(\varepsilon) \doteq \int_{0}^{1} R_{k}(\varepsilon) d k .
$$

The central planner maximizes present value of the expected flow of the political contributions $R$ from all regions $j \in[0,1]$ :

$$
G(R) \doteq E \int_{T}^{\infty} R e^{-\theta(\theta-T)} d \theta=\frac{R}{\rho} .
$$

Each region $j$ maximizes its expected utility $\Theta^{j}[$ cf. (23)].

According to Dixit at al. (1997), a subgame perfect Nash equilibrium for this lobbying game is a set of contribution schedules $R_{j}(\varepsilon)$ and a policy $\varepsilon$ such that the following conditions $(i)-(i v)$ hold: 
(i) Contributions $R_{j}$ are non-negative but no more than the contributor's income, $\Theta^{j} \geq 0$.

(ii) The policy $\varepsilon$ maximizes the central planner's welfare (26) taking the contribution schedules $R_{j}(\varepsilon)$ as given,

$$
\varepsilon=\arg \max _{\varepsilon} G(R(\varepsilon))=\arg \max _{\varepsilon \in[0,1]} R(\varepsilon)
$$

(iii) Region $j$ cannot have a feasible strategy $R_{j}(\varepsilon)$ that yields it a higher level of utility than in equilibrium, given the central planner's anticipated decision rule (14),

$$
\varepsilon=\arg \max _{\varepsilon} \Theta^{j}\left(m_{j}, m, R, R_{j}(\varepsilon), \gamma_{j}\right) \text { with } m_{j}=\varepsilon \widehat{m}_{j} \text { and } m=\varepsilon \widehat{m} \text {. }
$$

Because the region is small, it takes the total contributions of all regions, $R$, as given. However, the region observes the dependency of pollution $m$ on environmental policy $\varepsilon$ [cf. (14)].

(iv) Region $j$ provides the central planner at least with the level of utility than in the case it offers nothing $\left(R_{j}=0\right)$, and the central planner responds optimally given the other regions contribution functions,

$$
G(R(\varepsilon)) \geq\left.\max _{\varepsilon} G(R(\varepsilon))\right|_{R_{j}=0} .
$$

\subsection{The stationary state}

Noting (23), the conditions (28) for regions $j \in[0,1]$ is equivalent to

$$
\begin{aligned}
0 & =\frac{d \Theta^{j}}{d \varepsilon}=\frac{\partial \Theta^{j}}{\partial R_{j}} \frac{d R_{j}}{d \varepsilon}+\frac{\partial \Theta^{j}}{\partial m_{j}} \underbrace{\frac{\partial m_{j}}{\partial \varepsilon}}_{=\widehat{m}_{j}}+\frac{\partial \Theta^{j}}{\partial m} \underbrace{\frac{\partial m}{\partial \varepsilon}}_{=\widehat{m}}=\frac{\partial \Theta^{j}}{\partial R_{j}} \frac{d R_{j}}{d \varepsilon}+\frac{\partial \Theta^{j}}{\partial m_{j}} \widehat{m}_{j}+\frac{\partial \Theta^{j}}{\partial m} \widehat{m} \\
& =-\frac{m^{-\delta}}{\rho} \frac{d R_{j}}{d \varepsilon}+m^{-\delta} \Gamma^{j}\left[\xi^{j}\left(\frac{l_{j}}{m_{j}}\right)-\beta\right] \frac{\widehat{m}_{j}}{m_{j}}-\delta m^{-\delta}\left(\Gamma^{j}+\frac{R-R_{j}}{\rho}\right) \frac{\widehat{m}}{m}
\end{aligned}
$$


and

$$
\frac{1}{\rho} \frac{d R_{j}}{d \varepsilon}=\Gamma^{j}\left[\xi^{j}\left(\frac{l_{j}}{m_{j}}\right)-\beta\right] \frac{\widehat{m}_{j}}{m_{j}}-\delta\left(\Gamma^{j}+\frac{R-R_{j}}{\rho}\right) \frac{\widehat{m}}{m} \text { for } j \in[0,1] .
$$

Once the economy attains the stationary state, the emissions under the previous and current technology become equal: $\widehat{m}=m$ and $\widehat{m}_{j}=m_{j}$ for $j \in[0,1]$. Plugging these conditions into (29) yields

$$
\frac{1}{\rho} \frac{d R_{j}}{d \varepsilon}=\left[\xi^{j}\left(\frac{l_{j}}{m_{j}}\right)-\beta\right] \Gamma^{j}-\delta\left(\Gamma^{j}+\frac{R-R_{j}}{\rho}\right) \text { for } j \in[0,1] .
$$

Noting these equations and (25), the government's equilibrium condition (27) is equivalent to

$$
\begin{aligned}
0 & =\frac{d R}{d \varepsilon}=\int_{0}^{1} \frac{d R_{j}}{d \varepsilon} d j=\rho \int_{0}^{1}\left\{\left[\xi^{j}\left(\frac{l_{j}}{m_{j}}\right)-\beta\right] \Gamma^{j}-\delta\left(\Gamma^{j}+\frac{R-R_{j}}{\rho}\right)\right\} d j \\
& =\rho\{\int_{0}^{1}\left[\xi^{j}\left(\frac{l_{j}}{m_{j}}\right)-\beta-\delta\right] \Gamma^{j} d j-\frac{\delta}{\rho} \underbrace{\int_{0}^{1}\left(R-R_{j}\right) d j}_{=0}\} \\
& =\rho \int_{0}^{1}\left[\xi^{j}\left(\frac{l_{j}}{m_{j}}\right)-\beta-\delta\right] \Gamma^{j} d j .
\end{aligned}
$$

In the stationary state, all inputs $\left(l_{j}, m_{j}\right)$ for all regions $j \in[0,1]$ must be constant. Because the expected value of the flow of output, $\Gamma^{j}$ is a random variable for all regions $j \in[0,1]$, then, given (30), the only possible stationary state in the economy of regions $j \in[0,1]$ is

$$
\xi^{j}\left(\frac{l_{j}}{m_{j}}\right)=\beta+\delta \text { for } j \in[0,1] .
$$

This means that if region $j \in[0,1]$ has confidence on stable development, then it expects that its expenditure share of energy, $\xi^{j}$, will be equal to $\beta+\delta$ in the long run. From the equilibrium conditions (24) of the regions $j \in[0,1]$ as well as those (31) of the central planner, one obtains

$$
\xi^{j}\left(\frac{l^{G}}{m^{G}}\right)=\beta+\delta, \quad \frac{\left(a_{j}-1\right) \lambda_{j} l^{G}}{\rho+\left(1-a_{j}\right) \lambda_{j}\left(L-l^{G}\right)}=\alpha_{j}-\beta-\delta,
$$

where the superscript $G$ denotes grandfathering of emissions.

Comparing the systems (19) and (32) yields the following result: 
Proposition 4 Regulation leads to the Pareto optimum, $\left(l^{G}, m^{G}\right)=\left(l^{P}, m^{P}\right)$.

The introduction of a self-interested central planner has the same impact as that of a benevolent central planner: it internalizes the externality of emissions through pollution, leading to the Pareto optimum. This means that an agreement on a self-interested policy maker is self-enforcing: no region has incentives to break it.

\section{Conclusions}

This document examines the design of emission policy for a large number of regions which use land, labor and emitting inputs in production, but which can increase their total factor productivity by allocating labor to R\&D. The use of emitting inputs pollutes, decreasing welfare everywhere. The regions can agree on a central planner and authorize it to grant them GHG emission caps. Because the regions do not form a federation with a budget of its own, the central planner is non-benevolent, self-interested and subject to lobbying. It is plausible to assume that the policy parameter of the central planner is uniform throughout all regions.

By the use of grandfathering schemes with one policy parameter only, the central planner internalizes the negative externality through GHG emissions. When emission caps are set in proportion to past emissions, all regions face the same marginal benefits from emissions via allocation in subsequent periods. Because the basis for allocation is updated over time, the central planner has the full control of resources. Thus, an agreement on the central planner, benevolent or self-interested, leads to the first-best allocation of resources (i.e. the Pareto optimum). Consequently, that agreement is self-enforcing. 


\section{Appendix}

\section{A Proposition 1}

Region $j$ maximizes $(22)$ by $\left(l_{j}, m_{j}\right)$ subject to $(6)$, given $m$. It is equivalent to maximize

$$
E \int_{T}^{\infty} a_{j}^{\gamma_{j}} f^{j}\left(l_{j}, m_{j}\right) m_{j}^{-\beta} e^{-\rho(t-T)} d t
$$

by $\left(l_{j}, m_{j}\right)$ subject to $(6)$.

Assume for a while that energy input $m_{j}$ is kept constant. The value of this maximization is

$$
\Pi^{j}\left(\gamma_{j}, m_{j}, T\right)=\max _{l_{j} \text { s.t. }(6)} E \int_{T}^{\infty} a_{j}^{\gamma_{j}} f^{j}\left(l_{j}, m_{j}\right) m_{j}^{-\beta} e^{-\rho(t-T)} d t
$$

Let us denote $\Pi^{j}=\Pi^{j}\left(\gamma_{j}, m_{j}, T\right)$ and $\widetilde{\Pi}^{j}=\Pi^{j}\left(\gamma_{j}+1, m_{j}, T\right)$. The Bellman equation corresponding to the optimal program (33) is given by (cf. Dixit and Pindyck 1994)

$$
\begin{aligned}
& \rho \Pi^{j}=\max _{l_{j}, m_{j}} \Psi\left(l_{j}, m_{j}, \gamma_{j}, T\right), \quad \text { where } \\
& \Psi\left(l_{j}, m_{j}, \gamma_{j}, T\right)=a_{j}^{\gamma_{j}} f^{j}\left(l_{j}, m_{j}\right) m_{j}^{-\beta}+\left(\widetilde{\Pi^{j}}-\Pi^{j}\right) \lambda_{j}\left(L_{j}-l_{j}\right) .
\end{aligned}
$$

Noting (4), this leads to the first-order condition

$$
\begin{aligned}
\frac{\partial \Psi}{\partial l_{j}} & =a_{j}^{\gamma_{j}} f_{l}^{j}\left(l_{j}, m_{j}\right) m_{j}^{-\beta}-\lambda_{j}\left(\widetilde{\Pi^{j}}-\Pi^{j}\right) \\
& =\frac{1}{l_{j}} a_{j}^{\gamma_{j}} f^{j}\left(l_{j}, m_{j}\right) m_{j}^{-\beta}\left[1-\xi^{j}\left(\frac{l_{j}}{m_{j}}\right)\right]-\lambda_{j}\left(\widetilde{\Pi^{j}}-\Pi^{j}\right)=0 .
\end{aligned}
$$

To solve the dynamic program (33), assume that the value of the program, $\Pi^{j}$, is in fixed proportion $\vartheta_{j}>0$ to instantaneous utility at the optimum. Noting (4), this implies

$$
\begin{aligned}
& \Pi^{j}\left(\gamma_{j}, m_{j}, T\right)=\vartheta_{j} a_{j}^{\gamma_{j}} f^{j}\left(l_{j}^{*}, m_{j}\right) m_{j}^{-\beta} \text { with } \\
& \frac{\partial \Pi^{j}}{\partial m_{j}}=\Pi^{j}\left[\frac{f_{m}^{j}\left(l_{j}, m_{j}\right)}{f^{j}\left(l_{j}, m_{j}\right)}-\frac{\beta}{m_{j}}\right]=\frac{\Pi^{j}}{m_{j}}\left[\xi^{j}\left(\frac{l_{j}}{m_{j}}\right)-\beta\right],
\end{aligned}
$$


where $l_{j}^{*}$ is the optimal value of the control variable $l_{j}$. This implies

$$
\left(\widetilde{\Pi^{j}}-\Pi^{j}\right) / \Pi^{j}=a_{j}-1 .
$$

Inserting (36) and (37) into the Bellman equation (34) yields

$$
1 / \vartheta_{j}=\rho+\left(1-a_{j}\right) \lambda_{j}\left(L_{j}-l_{j}^{*}\right)>0
$$

Inserting (36), (37) and (38) into (35), and noting $\left(\xi^{j}\right)^{\prime}>0$ yield (12):

$$
\begin{aligned}
0 & =\vartheta_{j} \frac{l_{j}}{\Pi^{j}} \frac{\partial \Psi}{\partial l_{j}}=\underbrace{a_{j}^{\gamma_{j}} f^{j}\left(l_{j}, m_{j}\right) m_{j}^{-\beta} \frac{\vartheta_{j}}{\Pi^{j}}}_{=1}\left[\alpha_{j}-\xi^{j}\left(\frac{l_{j}}{m_{j}}\right)\right]-(\underbrace{\frac{\widetilde{\Pi^{j}}}{\Pi_{j}^{j}}}_{=a_{j}}-1) \lambda_{j} l_{j} \vartheta_{j} \\
& =\alpha_{j}-\xi^{j}\left(\frac{l_{j}}{m_{j}}\right)-\frac{\left(a_{j}-1\right) \lambda_{j} l_{j}}{\rho+\left(1-a_{j}\right) \lambda_{j}\left(L_{j}-l_{j}^{*}\right)}
\end{aligned}
$$

From (8), (33) and (38) it follows that

$$
\begin{aligned}
\Upsilon^{j} & =\max _{l_{j} \text { s.t. }(6)} E \int_{T}^{\infty} a_{j}^{\gamma_{j}} f^{j}\left(l_{j}, m_{j}\right) m_{j}^{-\beta} m^{-\delta} e^{-\rho(\theta-T)} d \theta \\
& =m^{-\delta} E \int_{T}^{\infty} a_{j}^{\gamma_{j}} f^{j}\left(l_{j}, m_{j}\right) m_{j}^{-\beta} e^{-\rho(\theta-T)} d \theta=m^{-\delta} \Pi^{j}\left(\gamma_{j}, m_{j}, T\right) .
\end{aligned}
$$

Results (36), (39) and (40) lead to Proposition 1.

\section{B Proposition 2}

Given (1), (3), (4) and (12), it then holds true that

$$
\begin{aligned}
& \rho+(\underbrace{1-a_{j}}_{-}) \lambda_{j}(\underbrace{L_{j}-l_{j}^{L}}_{+}) \underbrace{\xi^{j}}_{\in(0,1)}>\rho+\left(1-a_{j}\right) \lambda_{j}\left(L_{j}-l_{j}^{L}\right)>0, \\
& \frac{\left(a_{j}-1\right) \lambda_{j} l_{j}^{L}}{\rho+\left(1-a_{j}\right) \lambda_{j}\left(L_{j}-l_{j}^{L}\right)}<\alpha_{j}-\beta<\alpha_{j}<1, \quad \rho+\left(1-a_{j}\right) \lambda_{j} L_{j}>0 .
\end{aligned}
$$

Noting (1), (12) and (41) yield

$$
\begin{aligned}
& \frac{d}{d l_{j}^{L}} \log \left[\frac{\left(a_{j}-1\right) \lambda_{j} l_{j}^{L}}{\rho+\left(1-a_{j}\right) \lambda_{j}\left(L_{j}-l_{j}^{L}\right)}\right]=\frac{1}{l_{j}^{L}}[1-\underbrace{\frac{\left(a_{j}-1\right) \lambda_{j} l_{j}^{L}}{\rho+\left(1-a_{j}\right) \lambda_{j}\left(L_{j}-l_{j}^{L}\right)}}_{\in(0,1)}]>0 \text { and } \\
& \frac{d}{d l_{j}^{L}}\left[\frac{\left(a_{j}-1\right) \lambda_{j} l_{j}^{L}}{\rho+\left(1-a_{j}\right) \lambda_{j}\left(L_{j}-l_{j}^{L}\right)}\right]>0 .
\end{aligned}
$$


Noting this and differentiating the left-hand equation in (12), one obtains

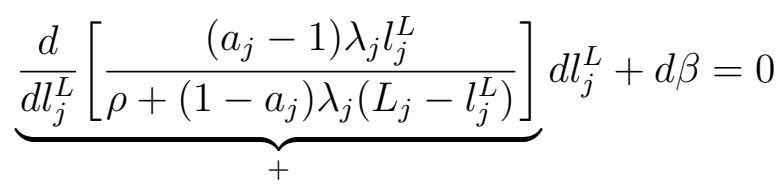

and $d l_{j}^{L} / d \beta<0$. Given (1), this implies $d z_{j}^{L} / d \beta=-d l_{j}^{L} / d \beta>0$. Finally, differentiating the right-hand equation in (12), and noting (12), one obtains

$$
\frac{d m_{j}^{L}}{d \beta}=\frac{m_{j}^{L}}{l_{j}^{L}}[\underbrace{\frac{d l_{j}^{L}}{d \beta}}_{-}-\underbrace{\frac{m_{j}^{L}}{\left(\xi^{j}\right)^{\prime}}}_{+}]<0 .
$$

\section{Function (23) and condition (24)}

Region $j$ maximizes $(22)$ by $l_{j}$ subject to $(6)$, given $\left(m, m_{j}, R, R_{j}\right)$. It is equivalent to maximize the expected value of the flow of output for region $j$,

$$
E \int_{T}^{\infty} a_{j}^{\gamma_{j}} f^{j}\left(l_{j}, m_{j}\right) m_{j}^{-\beta} e^{-\rho(\theta-T)} d \theta
$$

by $l_{j}$ subject to $(6)$, given $m_{j}$. The value of this maximization is

$$
\Gamma_{j}^{j}\left(\gamma_{j}, m_{j}, T\right)=\max _{l_{j} \text { s.t. }(6)} E \int_{T}^{\infty} a_{j}^{\gamma_{j}} f^{j}\left(l_{j}, m_{j}\right) m_{j}^{-\beta} e^{-\rho(\theta-T)} d \theta
$$

Denote $\Gamma^{j}=\Gamma^{j}\left(\gamma_{j}, m_{j}, T\right)$ and $\widetilde{\Gamma}^{j}=\Gamma^{j}\left(\gamma_{j}+1, m_{j}, T\right)$. The Bellman equation corresponding to the optimal program (42) is

$$
\begin{aligned}
& \rho \Gamma^{j}=\max _{l_{j}} \Psi\left(l_{j}, \gamma_{j}, m_{j}, R-R_{j}, T\right), \text { where } \\
& \Psi\left(l_{j}, \gamma_{j}, m_{j}, T\right)=a_{j}^{\gamma_{j}} f^{j}\left(l_{j}, m_{j}\right) m_{j}^{-\beta}+\lambda_{j}\left(L_{j}-l_{j}\right)\left(\widetilde{\Gamma}^{j}-\Gamma^{j}\right) .
\end{aligned}
$$

Noting (4), this leads to the first-order condition

$$
\begin{aligned}
\frac{\partial \Psi}{\partial l_{j}} & =a_{j}^{\gamma_{j}} f_{l}^{j}\left(l_{j}, m_{j}\right) m_{j}^{-\beta}-\lambda_{j}\left(\widetilde{\Gamma}^{j}-\Gamma^{j}\right) \\
& =\frac{1}{l_{j}} a_{j}^{\gamma_{j}} f^{j}\left(l_{j}, m_{j}\right) m_{j}^{-\beta}\left[\alpha_{j}-\xi^{j}\left(\frac{l_{j}}{m_{j}}\right)\right]-\lambda_{j}\left(\widetilde{\Gamma}^{j}-\Gamma^{j}\right)=0 .
\end{aligned}
$$


To solve the dynamic program (42), assume that the value of the program, $\Gamma^{j}$, is in fixed proportion $\vartheta_{j}>0$ to instantaneous utility:

$$
\Gamma^{j}\left(\gamma_{j}, m_{j}, T\right)=\vartheta_{j} a_{j}^{\gamma_{j}} f^{j}\left(l_{j}, m_{j}\right) m_{j}^{-\beta}
$$

where $l_{j}^{*}$ is the optimal value of the control variable $l_{j}$. This implies

$$
\left(\widetilde{\Gamma}^{j}-\Gamma^{j}\right) / \Gamma^{j}=a_{j}-1
$$

Inserting (48) and (46) into the Bellman equation (43) yields

$$
1 / \vartheta_{j}=\rho+\left(1-a_{j}\right) \lambda_{j}\left(L_{j}-l_{j}\right)>0 \text {. }
$$

Plugging this (47) into (45), one obtains

$$
\Gamma^{j}\left(\gamma_{j}, m_{j}, T\right)=\frac{a_{j}^{\gamma_{j}} f^{j}\left(l_{j}, m_{j}\right) m_{j}^{-\beta}}{\rho+\left(1-a_{j}\right) \lambda_{j}\left(L_{j}-l_{j}^{*}\right)},
$$

where $l_{j}^{*}$ - the optimal value of the control variable $l_{j}$ - is taken as given.

Inserting (48), (46) and (47) into (44), one obtains (24):

$$
\begin{aligned}
0 & =\vartheta_{j} \frac{l_{j}}{\Gamma^{j}} \frac{\partial \Psi}{\partial l_{j}}=\underbrace{a_{j}^{\gamma_{j}} f^{j}\left(l_{j}, m_{j}\right) m_{j}^{-\beta} \frac{\vartheta_{j}}{\Gamma^{j}}}_{=1}\left[\alpha_{j}-\xi^{j}\left(\frac{l_{j}}{m_{j}}\right)\right]-(\underbrace{\left.\frac{\widetilde{\Gamma}^{j}}{\Gamma^{j}}-1\right) \lambda_{j} l_{j} \vartheta_{j}}_{=a_{j}} \\
& =\alpha_{j}-\xi^{j}\left(\frac{l_{j}}{m_{j}}\right)-\frac{\left(a_{j}-1\right) \lambda_{j} l_{j}}{\rho+\left(1-a_{j}\right) \lambda_{j}\left(L_{j}-l_{j}\right)} .
\end{aligned}
$$

Noting (42) and (48), the expected utility (22) becomes (23):

$$
\begin{aligned}
& \Theta\left(m_{j}, m, R_{j}, R\right)=m^{-\delta} E \int_{T}^{\infty}\left[a_{j}^{\gamma_{j}} f^{j}\left(l_{j}, m_{j}\right) m_{j}^{-\beta}+R-R_{j}\right] e^{-\rho(\theta-T)} d \theta \\
& =m^{-\delta}\left[E \int_{T}^{\infty} a_{j}^{\gamma_{j}} f^{j}\left(l_{j}, m_{j}\right) m_{j}^{-\beta} e^{-\rho(\theta-T)} d \theta+\int_{T}^{\infty}\left(R-R_{j}\right) e^{-\rho(\theta-T)} d \theta\right] \\
& =m^{-\delta}\left[E \int_{T}^{\infty} a_{j}^{\gamma_{j}} f^{j}\left(l_{j}, m_{j}\right) m_{j}^{-\beta} e^{-\rho(\theta-T)} d \theta+\frac{R-R_{j}}{\rho}\right] \\
& =m^{-\delta}\left[\Gamma^{j}\left(\gamma_{j}, m_{j}, T\right)+\left(R-R_{j}\right) / \rho\right], \\
& \frac{\partial \Theta}{\partial m_{j}}=\frac{\Gamma^{j}}{m^{\delta}}\left[\frac{f_{m}^{j}\left(l_{j}, m_{j}\right)}{f^{j}\left(l_{j}, m_{j}\right)}-\frac{\beta}{m_{j}}\right]=\frac{\Gamma^{j}}{m^{\delta} m_{j}}\left[\xi^{j}\left(\frac{l_{j}}{m_{j}}\right)-\beta\right],
\end{aligned}
$$




$$
\partial \Theta / \partial M=-\delta m^{-\delta-1}\left[\Gamma^{j}+\left(R-R_{j}\right) / \rho\right], \quad-\partial \Theta / \partial R_{j}=\partial \Theta / \partial R=m^{-\delta} / \rho .
$$

\section{References:}

Aghion, P. and Howitt, P. (1998) Endogenous Growth Theory. MIT Press (Cambridge, Massachusetts, 1998).

Böhringer, Ch., and Lange, A. "On the design of optimal grandfathering schemes for emission allowances." European Economic Review, 49 (2005): 2041-2055.

Copeland, B.R. and Taylor, M.S. "Free trade and global warming: a trade theory view of the Kyoto protocol." Journal of Environmental Economics and Management, 49 (2005): 205-234.

Di Maria C. and Van der Werf, E. "Carbon leakage revisited: unilateral climate policy with directed technical change." Environmental and Resource Economics, 39 (2008): 55-74.

Dixit, A., Grossman, G.M. and Helpman, E. "Common agency and coordination: general theory and application to management policy making." Journal of Political Economy, 105 (1997): 752-769.

Dixit, A. and Pindyck, K. Investment under Uncertainty. Princeton University Press (Princeton, 1994).

Gerlach, R. and Kuik, O. "Carbon leakage with international technology spillovers." Fondazione Eni Enrico Mattei (2004): Paper 48.

Golombek, R. and Hoel, M. "Unilateral emission reductions and cross-region technological spillovers." Advances in Economic Analysis \& Policy, 4(2) (2004): Article 3.

Grossman, G.M. and Helpman, E. "Protection for sale." American Economic Review, 84 (1994): 833-850.

Haurie, A., Moresino, F., and Viguier, L. "A two-level differential game of international emissions trading." Advances in Dynamic Games. Annals of the International Society of Dynamic Games, 8 (2006), Part V: 293-307.

Jouvet, P.-A., Michel, P., and Rotillon, G. "The optimal initial allocation of pollution permits: a relative performance approach." Journal of Economic Dynamics and Control, 29 (2005): 1597-1609.

Palokangas, T. "International emission policy with lobbying and technological change." In: Crespo Cuaresma, J., Palokangas T. and Tarasyev, A. (eds.) Dynamic Systems, Economic Growth and the Environment. Springer Verlag (Heidelberg 2009). 
Xepapadeas, A. "Economic growth and the environment." In: Mähler K.-G,. and Vincent, J.R. (eds.) Handbook of Environmental Economics. Vol III. Elsevier (Amsterdam 2005): 1219-1271. 